\section{Excess weight and obesity prevalence in the RPS Brazilian Birth Cohort Consortium (Ribeirão Preto, Pelotas and São Luís)}

\author{
Prevalências de excesso de peso e obesidade no \\ Consórcio RPS (Ribeirão Preto, Pelotas e São Luís) \\ de coortes de nascimentos brasileiras, Brasil
}

\section{Prevalencias de sobrepeso y obesidad en el Consorcio RPS (Ribeirão Preto, Pelotas y São Luís) cohortes brasileñas de nascimientos}

Carolina Abreu de Carvalho 1

Elma Izze da Silva Magalhães 1

Heloisa Bettiol 2

Marco Antonio Barbieri 2

Viviane Cunha Cardoso 2

Alicia Matijasevich 3

Ana Maria Baptista Menezes 4

Bernardo Lessa Horta 4

Fernando C. Wehrmeister 4

Helen Gonçalves 4

Iná S. Santos 4

Natália Peixoto Lima 4

Ana Karina Teixeira da Cunha França 1

Antônio Augusto Moura da Silva 1

doi: 10.1590/0102-311X00237020

\begin{abstract}
Our objective was to estimate the prevalence of excess weight and obesity, according to sex and income in the RPS Brazilian Birth Cohort Consortium (Ribeirão Preto, Pelotas, and São Luís). Participants in the Ribeirão Preto (1978/1979 and 1994), Pelotas (1982, 1993 and 2004) and São Luís (1997/1998) birth cohorts were included in different follow-ups from 7 years old onwards. Excess weight (overweight and obesity) were assessed by body mass index. The highest prevalences were observed in Ribeirão Preto (excess weight: $27.7 \%$ at $9-11$ and $47.1 \%$ at 22-23 years; obesity: $8.6 \%$ at 9-11 and $19.8 \%$ at 22-23 years) while the smallest was in São Luís (excess weight: 5.4 to 7-9 and $17.2 \%$ at $18-19$ years; obesity: $1.8 \%$ at $7-9$ and $3.6 \%$ at $18-19$ years). The younger the cohort, the greater the prevalence of excess weight and obesity when comparing similar age groups. Increases in obesity prevalence were greater than in excess weight prevalence. Women had lower excess weight prevalence in older cohorts and higher obesity prevalence in younger cohorts. Higher excess weight and obesity prevalence were observed in higher income children and adolescents, and in poorer adults. Differences in the prevalence of excess weight and obesity evidenced that individuals from younger cohorts are more exposed to this morbidity, as well as those who were born in the most developed city, low-income adults as well as children and adolescents belonging to families of the highest income tertile. Therefore, the results of this study indicate the need to prioritize actions aimed at younger individuals.
\end{abstract}

Overweight; Obesity; Nutritional Transition; Prevalence

\author{
Correspondence \\ C. A. Carvalho \\ Programa de Pós-graduação em Saúde Coletiva, Universidade \\ Federal do Maranhão. \\ Rua Barão de Itapari 155, São Luís, MA 65020-070, Brasil. \\ carolina.carvalho@ufma.br

\footnotetext{
1 Universidade Federal do Maranhão, São Luís, Brasil. 2 Faculdade de Medicina de Ribeirão Preto, Universidade de São Paulo, São Paulo, Brsail

3 Faculdade de Medicina, Universidade de São Paulo, São Paulo, Brasil.

4 Faculdade de Medicina, Universidade Federal de Pelotas,
} \\ Pelotas, Brasil.
}




\section{Introduction}

The increase in overweight and obesity in the world is evident from the study carried out by the NCD Risk Factor Collaboration 1, with data from more than 19.2 million adults in 186 countries. According to this study, the prevalence of age-standardized obesity increased from 3.2\% in 1974 to $10.8 \%$ in 2014 among men, and from $6.4 \%$ to $14.9 \%$ among women. The same study estimates that in 2025 , the prevalence of obesity will reach $18 \%$ in men and exceed $21 \%$ in women. In Brazil, in 2018, the prevalence of excess weight among adults was $55.7 \%$, and of obesity was $19,8 \% 2$.

The main studies carried out in Brazil were cross-sectional 2,3, allowing to identify the prevalence of excess weight at different ages and to monitor its temporal trend. However, cohort studies initiated in different periods and regions make it possible to compare trends in excess weight in individuals from the same cohort over time and of similar age groups from cohorts that started in different periods and regions.

In a study conducted in the United States, Robinson et al. 4 identified that younger cohorts, born in the 1980s, were more prone to obesity than older ones, born in the 1960s, with more pronounced effects among women.

The RPS Brazilian Birth Cohort Consortium includes the three oldest research groups that have been following nine birth cohorts over a four-decade period. The cohorts involve three municipalities: Ribeirão Preto (São Paulo State), Pelotas (Rio Grande do Sul State) and São Luís (Maranhão State) from different regions of the country, using similar procedures for data collection 5.

In this context, our study aims to assess excess weight and obesity prevalence in six birth cohorts of the Brazilian cities of Ribeirão Preto, Pelotas and São Luís, at different ages, years of onset and follow-ups from the RPS Brazilian Birth Cohort Consortium. These prevalences will also be analyzed according to each individual's sex and income.

\section{Methods}

This is a study based on data from the RPS Brazilian Birth Cohort Consortium. This consortium is a research network involving three groups that develop the birth cohorts with the longest follow-up in Brazil, whose studies are national and international references in the life-cycle epidemiology.

Ribeirão Preto is located in the Southeastern Region of the country, northwest of the capital São Paulo. Its population in 2019 was 703,293 inhabitants 6 . According to the Brazilian Institute of Geography and Statistics (IBGE. https://cidades.ibge.gov.br/brasil/sp/ribeirao-preto/pesquisa/37/30255? tipo $=$ grafico\&localidade $1=431440 \&$ localidade $2=211130$, accessed on $28 /$ May $/ 2020$ ), the municipality's human development index (HDI) was 0.626 in 1991, 0.733 in 2000 and 0.800 in 2010.

Pelotas is located in the Extreme South of Brazil and, as of 2019, had 342,405 inhabitants 6. The HDI of this municipality in the years 1991, 2000 and 2010 was $0.558,0.660$ and 0.739 , respectively (IBGE. https://cidades.ibge.gov.br/brasil/sp/ribeirao-preto/pesquisa/37/30255?tipo=grafico\&locali dade $1=431440 \&$ localidade2 $=211130$, accessed on 28/May/2020).

São Luís, capital of the state of Maranhão, is located in the Northeastern region and in 2019 had a population of 1,101,884 inhabitants 6 . According to IBGE data (https://cidades.ibge.gov.br/bra sil/sp/ribeirao-preto/pesquisa/37/30255 tipo $=$ grafico\&localidade $1=431440 \&$ localidade $2=211130$, accessed on 28/May/2020) the HDI of that capital was 0.562 in 1991, 0.658 in 2000 and 0.768 in 2010.

This study includes six birth cohorts carried out in the cities of Ribeirão Preto (1978/1979 and 1994), Pelotas (1982, 1993 and 2004) and São Luís (1997/1998) and their respective followups: 1978/1979 Ribeirão Preto cohort - at 8-11 years (1987/1989), 23-25 years (2002/2004) and 37-39 years (2016/2017); 1994 Ribeirão Preto cohort - at 9-11 years (2004/2005) and 22-23 years (2016/2017); 1982 Pelotas cohort - at 18-19 (2000/2001), 22 (2004) and 30 years (2012); 1993 Pelotas cohort - at 11 years (2004), 18 years (2011) and 22 years (2015); 2004 Pelotas cohort - at 11 years (2015); and 1997/1998 São Luís cohort - at 7-9 years (2005/2006) and 18-19 years (2016/2017) (Supplementary Figure S1 - http://cadernos.ensp.fiocruz.br/static//arquivo/csp-2370-20-materialsuplementar_2577.pdf). Details on eligible, recruited and evaluated individuals in each follow-up are 
available elsewhere 8,9,10,11,12. Since not all cohorts followed individuals at the same age groups, in this study, comparisons between different cohorts were made considering close age groups.

The study outcome variables were excess weight and obesity. Excess weight included individuals classified as overweight and obese. As independent variables, sex and monthly family income were assessed.

The participants' weight was measured in kilograms $(\mathrm{kg})$ and the height was measured in centimeters $(\mathrm{cm})$. The equipment used in anthropometry was different between cohorts and/or follow-ups, as described below. In the 1978/1979 Ribeirão Preto cohort, for follow-ups at 8-11 and 23-25 years, and in the 1994 Ribeirão Preto cohort, for follow-up at 9-11 years, the weight was measured on Filizola (São Paulo, Brazil) mechanical scales, and the height in Formica-coated wooden stadiometers with 0.5 $\mathrm{cm}$ precision, built especially for this research.

In the Pelotas cohort (1982) at 18 years, electronic scales Tanita (Santo André, Brazil) and portable anthropometers CMS (Aparecida de Goiânia, Brazil) were used to measure weight and height, respectively. In the 1982 Pelotas cohort at 22 years and in the 1993 Pelotas cohort at 11 years, the weight was measured in Seca (Hamburg, Germany) electronic scales and the height in aluminum stadiometers with $0.1 \mathrm{~cm}$ precision, built for the research. In the 1997/1998 São Luís cohort at 7-9 years, a Plenna (São Paulo, Brazil) electronic scale was used to measure the weight and a portable AlturaExata (Belo Horizonte, Brazil) stadiometer to measure height. In the follow-up of the cohorts carried out since 2011, weight was obtained by a high precision scale coupled to an air displacement plethysmography device (BOD POD, Concord, United States) and height was measured with portable AlturaExata (Belo Horizonte, Brazil) stadiometers. In all cohorts and follow-ups, those anthropometric measurements were conducted following techniques recommended by the World Health Organization (WHO) 13.

The stages of the life course and the respective age ranges considered in the assessment of the nutritional status followed the definition used by the Brazilian Ministry of Health, recommended by the WHO. Thus, individuals under the age of 10 were considered as children; individuals between 10 and 19 years as adolescents, and those between 20 and 59 years as adults 14 .

Body mass index (BMI) was calculated for all individuals with available weight and height measurements, from the ratio between weight $(\mathrm{kg})$ and height $(\mathrm{m})$ squared. To classify the nutritional status of individuals under 18 years, the criteria established by Cole et al. ${ }^{15}$ was employed as it uses cutoff points that correspond to those proposed by the WHO to classify overweight and obesity in adulthood 16 . For individuals aged 18 years or older, the cutoff points proposed by the WHO 16 were employed.

In all cohorts, information on sex and family income was obtained through structured questionnaires applied by interviewers. Information about the child's sex was obtained shortly after birth, in the perinatal study. Family income was accounted for in monetary units or minimum wages and assessed in each cohort follow-up, with the exception of the 1982 Pelotas cohort at 18 years, in which the income of the men evaluated in the military recruitment was obtained during the data collection in $20018,9,10,11,12$.

Questionnaire application and anthropometric measurements were carried out by interviewers trained by the researchers, using standard techniques and periodically calibrated equipment 8,9,10,11,12. The study sample included all individuals with available weight and height data for calculating BMI in each of the follow-ups of the evaluated cohorts. The variables excess weight and obesity were categorized dichotomously (Yes/No) and family income was categorized into tertiles.

Statistical analysis was conducted using the Stata 14.0 software (https://www.stata.com). Prism 8.0 software (https://www.graphpad.com/scientific-software/prism/) was used to prepare the graphics. Excess weight and obesity prevalences and their respective $95 \%$ confidence intervals (95\% CI) were estimated for each of the follow-ups. In addition, crude prevalence ratios and their $95 \% \mathrm{CI}$ for excess weight and obesity were calculated to compare the differences between follow-ups at different ages in the same cohort, using mixed effects Poisson regression. In other comparisons (between different cohorts from the same city in similar age ranges, between different cohorts from different cities in similar age ranges, between cohorts from different cities in similar age ranges and follow-ups of different cohorts at the same or close years, according to sex and family income in tertiles), Poisson regression with robust variance estimates was used to estimate crude prevalence ratios and their $95 \%$ CI. 
Individuals with missing data on family income were excluded from the analyses stratified by income. Inverse probability weighting was used to correct estimates considering follow-up losses. Weighted estimates were used when the difference between weighted and unweighted estimates was greater than $0.5 \%$.

The research was approved by the Human Ethics Research Committees of the Ribeirão Preto Medical School of the University of São Paulo, the Medical School of the Federal University of Pelotas and the Presidente Dutra University Hospital of the Federal University of Maranhão. It was conducted in accordance with the ethical standards set out in the 1964 Helsinki Declaration and its subsequent amendments. The informed consent form was signed by the study participants or their parents/ guardians, except in the first follow-ups of the oldest cohorts, when only verbal consent was obtained.

\section{Results}

This study included participants from the RPS Brazilian Birth Cohort Consortium followed at specific age ranges, who had data on weight and height.

In the 1978/1979 Ribeirão Preto cohort, among the selected individuals, 2,893 participants from 8-11 years, 2,096 from 23-25 years, and 1,604 from 37-39 years with weight and height data for BMI calculation were included in this study.

Regarding losses, in the follow-ups at 8-11, 23-25 and 37-39 years of age of the 1978/1979 Ribeirão Preto birth cohort, a higher percentage of individuals with greater weight and length at birth that were born to married women attended the follow-ups. In the 8-11- and 23-25-year follow-ups, a higher percentage of individuals with family income above 3 minimum wages were monitored, but a lower percentage of those were born to adolescent mothers, with up to four years of schooling and with a higher number of children participating in the follow-ups. In the 23-25- and 37-39-year follow-ups, a higher percentage of women were monitored, whereas at 37-39 years of age, a higher percentage of individuals with family income above 1 minimum wage and born to mothers who had not studied attended the follow-up.

In the second Ribeirão Preto cohort (1994), the total number of individuals who presented information on weight and height for inclusion in the study was 788 at the age range of 9-11 years, and 618 at $22-23$ years.

There were sampling losses during the follow-up of the 1994 Ribeirão Preto cohort. From 22-23 years, individuals who attended the follow-up were mostly females, those with higher average birth weight, higher maternal age, from mothers with a partner, from families with lower income, and from mothers who did study.

In the first Pelotas cohort (1982), from the individuals evaluated, 3,147 at the age of 18-19, 4,288 at 22 years, and 3,551 at 30 years had data on weight and height and were included in this study.

In the second Pelotas cohort (1993), at 18 years, 4,106 adolescents were interviewed. Finally, at 22 years, 3,810 individuals were evaluated. This study included 4,441 individuals at the age of 11, 3,974 at 18 years and 3,591 at 22 years, with complete data on weight and height.

In the third Pelotas cohort (2004), at 11 years, 3,566 individuals were interviewed, of which 3,493 had information on weight and height.

In the São Luís cohort (1997/1998), among the individuals evaluated, 672 at 7-9 years and 610 at 18-19 years had available data on weight and height for inclusion in this study.

As described, there were losses of participants during the follow-ups, which occurred due to nonlocation of the individual, changes of address, deaths, and refusals.

For the family income variable, some follow-ups presented participants with missing data, with the lowest and highest percentage of information losses observed at the 22 years follow-up of the Pelotas cohort (1982) (0.2\%) and at the 7-9 years follow-up of the São Luís cohort (16.5\%), respectively. 


\section{Prevalence of excess weight and obesity - Ribeirão Preto cohorts}

In the oldest cohort (1978/1979), excess weight prevalence increased 2.27 times (95\%CI: 2.07-2.50), from 8-11 to 23-25 years. Regarding obesity, this increase was even more significant $(\mathrm{PR}=2.91$; 95\%CI: 2.40-3.53). From 23-25 to 37-39 years, the prevalence of excess weight doubled ( $\mathrm{PR}=2.06$; 95\%CI: 1.95-2.18) and the prevalence of obesity almost tripled ( $\mathrm{PR}=2.95$; 95\%CI: 2.62-3.33). At 37-39 years, 74.2\% had excess weight and 35\% were obese (Table 1 and Figure 1; data from PR and 95\%CI not shown in table).

In the youngest cohort (1994), from 9-11 years to 22-23 years, the increase in excess weight prevalence was 1.76 times (95\%CI: 1.55-1.99), and the increase in obesity, 2.5 times (95\%CI: 1.94-3.21) (Table 1 and Figure 1; data from PR and 95\%CI not shown in table).

At 8-11 years, there were higher prevalences of excess weight and obesity in the youngest than in the oldest cohort. The same was observed at 22-25 years for excess weight and obesity. However, the increase in prevalence from 8-11 to 22-25 years was smaller in the youngest cohort (Table 2).

In the 1978/1979 cohort, a lower excess weight prevalence was observed in females than in males at 23-25 years and at 37-39 years. No differences were observed in the prevalence of obesity in relation to sex in any of the follow-ups (Table 3).

In the 1994 cohort, the prevalence of excess weight did not differ significantly between the sexes, in any of the follow-ups. However, only among 22-23 years, obesity prevalence was $51 \%$ higher in females compared to males (Table 3).

Regarding income, in both cohorts at 8-11 years, there was a higher prevalence of excess weight among individuals from families in the third income tertile when compared to those in the first tertile. On the other hand, in the 1978/1979 cohort at 23-25 years, lower prevalences of excess weight and obesity were found among individuals in the third tertile compared to those in the first income tertile (Table 4).

Table 1

Excess weight and obesity prevalence in RPS Brazilian Birth Cohort Consortium (Ribeirão Preto, Pelotas and São Luís).

\begin{tabular}{|c|c|c|c|c|c|c|}
\hline \multirow[t]{2}{*}{ Cohorts/Age (years) } & \multirow[t]{2}{*}{ Year of follow-up } & \multirow[t]{2}{*}{$\mathbf{n}$} & \multicolumn{2}{|c|}{ Excess weight * } & \multicolumn{2}{|c|}{ Obesity } \\
\hline & & & $\%$ & $95 \% \mathrm{Cl}$ & $\%$ & $95 \% \mathrm{Cl}$ \\
\hline \multicolumn{7}{|c|}{ Ribeirão Preto (1978/1979) } \\
\hline $8-11$ & $1987 / 1989$ & 2,893 & 14.8 & $13.5-16.2$ & 3.9 & $3.2-4.7$ \\
\hline $23-25$ & $2002 / 2004$ & 2,096 & 36.5 & $34.4-38.7$ & 12.2 & 10.7-13.7 \\
\hline $37-39$ & $2016 / 2017$ & 1,595 & 74.2 & $71.8-76.4$ & 35.0 & $32.6-37.5$ \\
\hline \multicolumn{7}{|l|}{ Ribeirão Preto (1994) } \\
\hline $9-11$ & $2004 / 2005$ & 788 & 27.7 & 24.6-31.1 & 8.6 & $6.7-10.8$ \\
\hline $22-23$ & $2016 / 2017$ & 618 & 47.1 & $42.5-51.7$ & 19.8 & 16.4-23.7 \\
\hline \multicolumn{7}{|l|}{ Pelotas (1982) } \\
\hline $18-19$ & $2000 / 2001$ & 3,147 & 17.9 & 16.6-19.3 & 4.8 & $4.1-5.6$ \\
\hline 22 & 2004 & 4,288 & 28.9 & $27.5-30.3$ & 8.1 & 7.3-9.0 \\
\hline 30 & 2012 & 3,551 & 57.5 & 55.9-59.1 & 22.9 & $21.5-24.3$ \\
\hline \multicolumn{7}{|l|}{ Pelotas (1993) } \\
\hline 11 & 2004 & 4,441 & 24.3 & 23.0-25.6 & 6.0 & $5.3-6.7$ \\
\hline 18 & 2011 & 3,974 & 27.6 & 26.2-29.0 & 9.1 & $8.2-10.0$ \\
\hline 22 & 2015 & 3,591 & 44.2 & $42.6-45.9$ & 16.9 & 15.7-18.1 \\
\hline \multicolumn{7}{|l|}{ Pelotas (2004) } \\
\hline 11 & 2015 & 3,493 & 37.2 & $35.6-38.8$ & 13.2 & $12.1-14.3$ \\
\hline \multicolumn{7}{|l|}{ São Luís (1997/1998) } \\
\hline $7-9$ & $2005 / 2006$ & 672 & 5.4 & $3.9-7.5$ & 1.8 & $1.0-3.2$ \\
\hline $18-19$ & $2016 / 2017$ & 610 & 17.2 & $14.3-20.4$ & 3.6 & $2.3-5.4$ \\
\hline
\end{tabular}

95\%Cl: 95\% confidence interval.

* Includes overweight and obesity. 


\section{Figure 1}

Prevalence of excess weight and obesity in RPS Brazilian Birth Cohort Consortium (Ribeirão Preto, Pelotas and São Luís), by age.

1a) Ribeirão Preto


Age (years)

1b) Pelotas
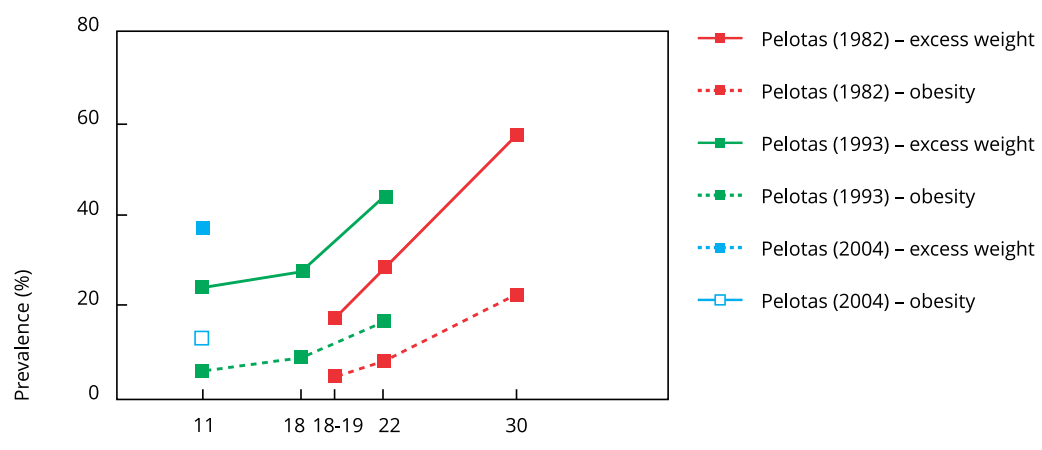

Age (years)

1c) São Luís

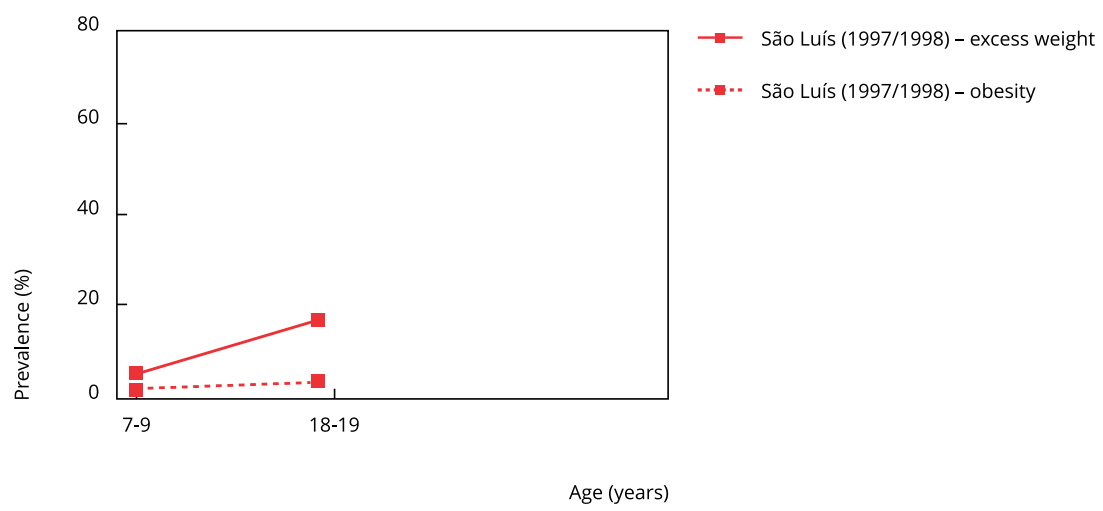


Table 2

Comparisons of excess weight and obesity prevalence in RPS Brazilian Birth Cohort Consortium (Ribeirão Preto, Pelotas and São Luís).

\begin{tabular}{|c|c|c|c|c|c|}
\hline \multirow[t]{2}{*}{ Cities/age range/cohorts } & \multirow[t]{2}{*}{ Total } & \multicolumn{2}{|c|}{ Excess weight * } & \multicolumn{2}{|c|}{ Obesity } \\
\hline & & n (\%) & Crude PR $(95 \% \mathrm{Cl})$ & n (\%) & Crude PR $(95 \% \mathrm{Cl})$ \\
\hline \multicolumn{6}{|c|}{ Cohorts from the same city in similar age ranges } \\
\hline \multicolumn{6}{|l|}{ From 8 to 11 years } \\
\hline Ribeirão Preto (1978/1979) - 8-11 years & 2,893 & $428(14.8)$ & 1.00 & $113(3.9)$ & 1.00 \\
\hline Ribeirão Preto (1994) - 9-11 years & 788 & $218(27.7)$ & $1.87(1.62-2.16)$ & $68(8.6)$ & $2.21(1.65-2.95)$ \\
\hline \multicolumn{6}{|l|}{ From 22 to 25 years } \\
\hline Ribeirão Preto (1978/1979) - 23-25 years & 2,096 & $765(36.5)$ & 1.00 & $256(12.2)$ & 1.00 \\
\hline Ribeirão Preto (1994) - 22-23 years & 553 & $260(47.1)$ & $1.29(1.16-1.43)$ & 109 (19.8) & $1.62(1.32-1.98)$ \\
\hline \multicolumn{6}{|l|}{ Up to 11 years } \\
\hline Pelotas (1993) - 11 years & 4,441 & $1,079(24.3)$ & 1.00 & $266(6.0)$ & 1.00 \\
\hline Pelotas (2004) - 11 years & 3,493 & $1,299(37.2)$ & $1.53(1.43-1.64)$ & $461(13.2)$ & $2.20(1.91-2.55)$ \\
\hline \multicolumn{6}{|l|}{ From 18 to 19 years } \\
\hline Pelotas (1982) - $18-19$ years & 3,147 & $563(17.9)$ & 1.00 & $151(4.8)$ & 1.00 \\
\hline Pelotas (1993) - 18 years & 3,974 & $1,097(27.6)$ & $1.54(1.41-1.69)$ & $362(9.1)$ & $1.90(1.58-2.28)$ \\
\hline \multicolumn{6}{|l|}{ Up to 22 years } \\
\hline Pelotas (1982) - 22 years & 4,288 & $1,239(28.9)$ & 1.00 & $347(8.1)$ & 1.00 \\
\hline Pelotas (1993) - 22 years & 3,591 & $1,587(44.2)$ & $1.53(1.44-1.62)$ & $607(16.9)$ & $2.09(1.85-2.37)$ \\
\hline \multicolumn{6}{|c|}{$\begin{array}{l}\text { Different cohorts from different cities in similar } \\
\text { age ranges }\end{array}$} \\
\hline \multicolumn{6}{|l|}{ From 8 to 11 years } \\
\hline Ribeirão Preto (1978/1979) - 8-11 years & 2,893 & $428(14.8)$ & 1.00 & $113(3.9)$ & 1.00 \\
\hline Pelotas (1993) - 11 years & 4,441 & $1,079(24.3)$ & $1.64(1.48-1.82)$ & $266(6.0)$ & $1.54(1.24-1.90)$ \\
\hline Ribeirão Preto (1994) - 9-11 years & 788 & $218(27.7)$ & $1.87(1.62-2.16)$ & $68(8.6)$ & $2.21(1.65-2.95)$ \\
\hline São Luís (1997) - 7-9 years & 672 & $36(5.4)$ & $0.36(0.26-0.50)$ & $12(1.8)$ & $0.46(0.25-0.82)$ \\
\hline Pelotas (2004) - 11 years & 3,493 & $1,299(37.2)$ & $2.51(2.28-2.77)$ & $461(13.2)$ & $3.38(2.77-4.13)$ \\
\hline \multicolumn{6}{|l|}{ From 18 to 19 years } \\
\hline Pelotas (1982) - $18-19$ years & 3,147 & $563(17.9)$ & 1.00 & $151(4.8)$ & 1.00 \\
\hline Pelotas (1993) - 18 years & 3,974 & $1,097(27.6)$ & $1.54(1.41-1.69)$ & $362(9.1)$ & $1.90(1.58-2.28)$ \\
\hline São Luís (1997/1998) - 18-19 years & 610 & $105(17.2)$ & $0.96(0.80-1.16)$ & $22(3.6)$ & $0.75(0.48-1.17)$ \\
\hline \multicolumn{6}{|l|}{ From 22 to 25 years } \\
\hline Ribeirão Preto (1978/1979) - 23-25 years & 2,096 & $765(36.5)$ & 1.00 & $256(12.2)$ & 1.00 \\
\hline Pelotas (1982) - 22 years & 4,288 & $1,239(28.9)$ & $0.79(0.74-0.85)$ & $347(8.1)$ & $0.66(0.57-0.77)$ \\
\hline Pelotas (1993) - 22 years & 3,591 & $1,587(44.2)$ & $1.21(1.13-1.30)$ & $607(16.9)$ & $1.39(1.21-1.59)$ \\
\hline Ribeirão Preto (1994) - 22-23 years & 553 & $260(47.1)$ & $1.29(1.16-1.43)$ & 109 (19.8) & $1.62(1.32-1.98)$ \\
\hline \multicolumn{6}{|l|}{ Cohorts from different cities in similar age } \\
\hline \multicolumn{6}{|l|}{ ranges and similar follow-up years } \\
\hline \multicolumn{6}{|l|}{ From 8 to 11 years in $2004 / 2006$} \\
\hline Pelotas (1993) - 11 years & 4,441 & $1,079(24.3)$ & 1.00 & $266(6.0)$ & 1.00 \\
\hline Ribeirão Preto (1994) - 9-11 years & 788 & $218(27.7)$ & $1.14(1.01-1.29)$ & $68(8.6)$ & $1.43(1.12-1.86)$ \\
\hline São Luís (1997) - 7-9 years & 672 & $36(5.4)$ & $0.22(0.16-0.30)$ & $12(1.8)$ & $0.30(0.17-0.53)$ \\
\hline \multicolumn{6}{|l|}{ From 18 to 23 years in 2015/2017 } \\
\hline Pelotas (1993) - 22 years & 3,591 & $1,587(44.2)$ & 1.00 & 607 (16.9) & 1.00 \\
\hline Ribeirão Preto (1994) - 22-23 years & 553 & $260(47.1)$ & $1.07(1.01-1.17)$ & $109(19.8)$ & $1.17(1.07-1.40)$ \\
\hline São Luís (1997/1998) - 18-19 years & 610 & $105(17.2)$ & $0.39(0.33-0.47)$ & $22(3.6)$ & $0.21(0.14-0.32)$ \\
\hline
\end{tabular}

95\%Cl: 95\% confidence interval; PR: prevalence ratio.

* Includes overweight and obesity. 
Table 3

Excess weight and obesity prevalence in RPS Brazilian Birth Cohort Consortium (Ribeirão Preto, Pelotas and São Luís), by sex.

\begin{tabular}{|c|c|c|c|c|c|c|c|c|}
\hline \multirow[t]{2}{*}{ Cohorts/Age (years) } & \multirow{2}{*}{$\begin{array}{c}\text { Year of } \\
\text { follow-up }\end{array}$} & \multirow[t]{2}{*}{$\mathbf{n}$} & \multicolumn{3}{|c|}{ Excess weight * } & \multicolumn{3}{|c|}{ Obesity } \\
\hline & & & Male ** $(\%)$ & Female (\%) & $\begin{array}{c}\text { Crude PR } \\
(95 \% \mathrm{Cl})\end{array}$ & Male ** (\%) & Female (\%) & $\begin{array}{c}\text { Crude PR } \\
(95 \% \mathrm{Cl})\end{array}$ \\
\hline \multicolumn{9}{|c|}{ Ribeirão Preto (1978/1979) } \\
\hline $8-11$ & 1987/1989 & 2,893 & 13.5 & 16.2 & $\begin{array}{c}1.20 \\
(1.00-1.43)\end{array}$ & 4.4 & 3.3 & $\begin{array}{c}0.75 \\
(0.51-1.08)\end{array}$ \\
\hline $23-25$ & $2002 / 2004$ & 2,096 & 43.5 & 29.3 & $\begin{array}{c}0.67 \\
(0.60-0.76)\end{array}$ & 12.9 & 11.4 & $\begin{array}{c}0.88 \\
(0.69-1.12)\end{array}$ \\
\hline $37-39$ & $2016 / 2017$ & 1,595 & 79.8 & 68.3 & $\begin{array}{c}0.86 \\
(0.81-0.91)\end{array}$ & 35.6 & 34.3 & $\begin{array}{c}0.96 \\
(0.84-1.11)\end{array}$ \\
\hline \multicolumn{9}{|l|}{ Ribeirão Preto (1994) } \\
\hline $9-11$ & $2004 / 2005$ & 788 & 27.6 & 27.8 & $\begin{array}{c}1.01 \\
(0.80-1.28)\end{array}$ & 8.3 & 8.8 & $\begin{array}{c}1.06 \\
(0.66-1.71)\end{array}$ \\
\hline $22-23$ & $2016 / 2017$ & 618 & 45.3 & 48.9 & $\begin{array}{c}1.08 \\
(0.89-1.31)\end{array}$ & 15.8 & 23.9 & $\begin{array}{c}1.51 \\
(1.02-2.24)\end{array}$ \\
\hline \multicolumn{9}{|l|}{ Pelotas (1982) } \\
\hline $18-19$ & $2000 / 2001$ & 3,147 & 17.2 & 19.5 & $\begin{array}{c}1.13 \\
(0.96-1.33)\end{array}$ & 4.5 & 5.4 & $\begin{array}{c}1.20 \\
(0.87-1.69)\end{array}$ \\
\hline 22 & 2004 & 4,288 & 30.6 & 27.0 & $\begin{array}{c}0.88 \\
(0.80-0.97)\end{array}$ & 7.5 & 9.1 & $\begin{array}{c}1.21 \\
(0.99-1.48)\end{array}$ \\
\hline 30 & 2012 & 3,551 & 62.8 & 52.3 & $\begin{array}{c}0.83 \\
(0.79-0.88)\end{array}$ & 22.1 & 23.8 & $\begin{array}{c}1.08 \\
(0.95-1.21)\end{array}$ \\
\hline \multicolumn{9}{|l|}{ Pelotas (1993) } \\
\hline 11 & 2004 & 4,441 & 24.5 & 24.0 & $\begin{array}{c}0.98 \\
(0.88-1.08)\end{array}$ & 6.4 & 5.6 & $\begin{array}{c}0.88 \\
(0.70-1.11)\end{array}$ \\
\hline 18 & 2011 & 3,974 & 26.4 & 28.7 & $\begin{array}{c}1.09 \\
(0.99-1.21)\end{array}$ & 8.1 & 10.0 & $\begin{array}{c}1.23 \\
(1.01-1.51)\end{array}$ \\
\hline 22 & 2015 & 3,591 & 44.0 & 44.4 & $\begin{array}{c}1.01 \\
(0.94-1.09)\end{array}$ & 14.0 & 19.4 & $\begin{array}{c}1.39 \\
(1.19-1.61)\end{array}$ \\
\hline \multicolumn{9}{|l|}{ Pelotas (2004) } \\
\hline 11 & 2015 & 3,493 & 37.3 & 37.0 & $\begin{array}{c}0.99 \\
(0.91-1.08)\end{array}$ & 13.0 & 13.3 & $\begin{array}{c}1.02 \\
(0.87-1.22)\end{array}$ \\
\hline \multicolumn{9}{|l|}{ São Luís (1997/1998) } \\
\hline $7-9$ & $2005 / 2006$ & 672 & 5.0 & 5.9 & $\begin{array}{c}1.18 \\
(0.62-2.27)\end{array}$ & 2.6 & 1.0 & $\begin{array}{c}0.38 \\
(0.11-1.38)\end{array}$ \\
\hline $18-19$ & $2016 / 2017$ & 610 & 17.1 & 17.4 & $\begin{array}{c}1.02 \\
(0.71-1.46)\end{array}$ & 3.4 & 3.9 & $\begin{array}{c}1.15 \\
(0.50-2.64)\end{array}$ \\
\hline
\end{tabular}

95\%Cl: 95\% confidence interval; PR: prevalence ratio.

* Includes overweight and obesity;

** Reference category. 
Table 4

Excess weight and obesity prevalences in RPS Brazilian Birth Cohort Consortium (Ribeirão Preto, Pelotas and São Luís), by income tertiles.

\begin{tabular}{|c|c|c|c|c|c|c|c|c|c|c|c|c|}
\hline \multirow{3}{*}{ Cohorts/Age (years) } & \multirow{3}{*}{$\begin{array}{c}\text { Year of } \\
\text { follow-up }\end{array}$} & \multirow{3}{*}{$\mathbf{n}$} & \multicolumn{5}{|c|}{ Excess weight * } & \multicolumn{5}{|c|}{ Obesity } \\
\hline & & & \multirow{2}{*}{$\begin{array}{c}\text { 1st tertile ** } \\
\%\end{array}$} & \multicolumn{2}{|c|}{ 2nd tertile } & \multicolumn{2}{|c|}{ 3rd tertile } & \multirow{2}{*}{$\begin{array}{c}\text { 1st tertile ** } \\
\%\end{array}$} & \multicolumn{2}{|c|}{ 2nd tertile } & \multicolumn{2}{|r|}{ 3rd tertile } \\
\hline & & & & $\%$ & $\begin{array}{c}\text { Crude PR } \\
(95 \% \mathrm{Cl})\end{array}$ & $\%$ & $\begin{array}{l}\text { Crude PR } \\
(95 \% \mathrm{Cl})\end{array}$ & & $\%$ & $\begin{array}{c}\text { Crude PR } \\
(95 \% \mathrm{Cl})\end{array}$ & $\%$ & $\begin{array}{c}\text { Crude PR } \\
(95 \% \mathrm{Cl})\end{array}$ \\
\hline \multicolumn{13}{|l|}{$\begin{array}{l}\text { Ribeirão Preto } \\
(1978 / 1979)\end{array}$} \\
\hline $8-11$ & 1987/1989 & 2,432 & 10.8 & 13.4 & $\begin{array}{c}1.24 \\
(0.93-1.64)\end{array}$ & 23.1 & $\begin{array}{c}2.14 \\
(1.69-2.80)\end{array}$ & 3.4 & 3.6 & $\begin{array}{c}1.06 \\
(0.64-1.90)\end{array}$ & 4.9 & $\begin{array}{c}1.44 \\
(0.93-2.56)\end{array}$ \\
\hline $23-25$ & $2002 / 2004$ & 1,938 & 39.0 & 35.6 & $\begin{array}{c}0.91 \\
(0.81-1.08)\end{array}$ & 31.8 & $\begin{array}{c}0.82 \\
(0.71-0.98)\end{array}$ & 15.2 & 12.3 & $\begin{array}{c}0.81 \\
(0.63-1.10)\end{array}$ & 8.0 & $\begin{array}{c}0.53 \\
(0.37-0.74)\end{array}$ \\
\hline $37-39$ & $2016 / 2017$ & 1,604 & 74.9 & 73.6 & $\begin{array}{c}0.98 \\
(0.91-1.06)\end{array}$ & 74.3 & $\begin{array}{c}0.99 \\
(0.92-1.07)\end{array}$ & 36.4 & 36.9 & $\begin{array}{c}1.01 \\
(0.85-1.21)\end{array}$ & 32.2 & $\begin{array}{c}0.88 \\
(0.74-1.05)\end{array}$ \\
\hline \multicolumn{13}{|l|}{ Ribeirão Preto (1994) } \\
\hline $9-11$ & $2004 / 2005$ & 556 & 3.6 & 25.7 & $\begin{array}{c}1.09 \\
(0.76-1.57)\end{array}$ & 38.3 & $\begin{array}{c}1.62 \\
(1.16-2.28)\end{array}$ & 7.5 & 8.0 & $\begin{array}{c}1.07 \\
(0.51-2.21)\end{array}$ & 13.0 & $\begin{array}{c}1.73 \\
(0.88-3.43)\end{array}$ \\
\hline $22-23$ & $2016 / 2017$ & 618 & 45.5 & 51.1 & $\begin{array}{c}1.12 \\
(0.93-1.40)\end{array}$ & 44.9 & $\begin{array}{c}0.99 \\
(0.82-1.27)\end{array}$ & 21.0 & 23.9 & $\begin{array}{c}1.14 \\
(0.77-1.63)\end{array}$ & 14.9 & $\begin{array}{c}0.71 \\
(0.57-1.29)\end{array}$ \\
\hline \multicolumn{13}{|l|}{ Pelotas (1982) } \\
\hline $18-19$ & $2000 / 2001$ & 1,555 & 17.2 & 18.5 & $\begin{array}{c}1.08 \\
(0.83-1.40)\end{array}$ & 19.1 & $\begin{array}{c}1.11 \\
(0.86-1.44)\end{array}$ & 3.9 & 5.3 & $\begin{array}{c}1.36 \\
(0.79-2.42)\end{array}$ & 4.5 & $\begin{array}{c}1.15 \\
(0.65-2.09)\end{array}$ \\
\hline 22 & 2004 & 4,288 & 29.0 & 29.5 & $\begin{array}{c}1.02 \\
(0.91-1.14)\end{array}$ & 28.0 & $\begin{array}{c}0.97 \\
(0.86-1.08)\end{array}$ & 9.5 & 8.8 & $\begin{array}{c}0.93 \\
(0.73-1.17)\end{array}$ & 6.7 & $\begin{array}{c}0.71 \\
(0.55-0.91)\end{array}$ \\
\hline 30 & 2012 & 3,358 & 55.7 & 61.5 & $\begin{array}{c}1.10 \\
(1.03-1.18)\end{array}$ & 56.5 & $\begin{array}{c}1.01 \\
(0.94-1.09)\end{array}$ & 24.0 & 23.3 & $\begin{array}{c}0.97 \\
(0.84-1.13)\end{array}$ & 21.7 & $\begin{array}{c}0.90 \\
(0.78-1.05)\end{array}$ \\
\hline \multicolumn{13}{|l|}{ Pelotas (1993) } \\
\hline 11 & 2004 & 4,441 & 18.2 & 24.6 & $\begin{array}{c}1.35 \\
(1.18-1.55)\end{array}$ & 30.1 & $\begin{array}{c}1.65 \\
(1.45-1.89)\end{array}$ & 4.5 & 6.1 & $\begin{array}{c}1.36 \\
(0.99-1.83)\end{array}$ & 7.4 & $\begin{array}{c}1.64 \\
(1.21-2.20)\end{array}$ \\
\hline 18 & 2011 & 3,974 & 26.4 & 28.2 & $\begin{array}{c}1.07 \\
(0.94-1.21)\end{array}$ & 28.1 & $\begin{array}{c}1.06 \\
(0.94-1.20)\end{array}$ & 9.2 & 9.2 & $\begin{array}{c}1.00 \\
(0.78-1.26)\end{array}$ & 8.9 & $\begin{array}{c}0.97 \\
(0.76-1.22)\end{array}$ \\
\hline 22 & 2015 & 3,402 & 44.2 & 46.1 & $\begin{array}{c}1.04 \\
(0.95-1.14)\end{array}$ & 42.8 & $\begin{array}{c}0.97 \\
(0.88-1.06)\end{array}$ & 19.2 & 17.7 & $\begin{array}{c}0.92 \\
(0.78-1.10)\end{array}$ & 14.6 & $\begin{array}{c}0.76 \\
(0.63-0.92)\end{array}$ \\
\hline \multicolumn{13}{|l|}{ Pelotas (2004) } \\
\hline 11 & 2015 & 3,493 & 32.9 & 37.6 & $\begin{array}{c}1.14 \\
(1.02-1.27)\end{array}$ & 41.1 & $\begin{array}{c}1.25 \\
(1.12-1.39)\end{array}$ & 13.5 & 13.6 & $\begin{array}{c}1.01 \\
(0.82-1.24)\end{array}$ & 12.5 & $\begin{array}{c}0.93 \\
(0.75-1.14)\end{array}$ \\
\hline \multicolumn{13}{|l|}{ São Luís (1997/1998) } \\
\hline $7-9$ & $2005 / 2006$ & 627 & 2.5 & 5.7 & $\begin{array}{c}2.28 \\
(0.82-6.17)\end{array}$ & 7.9 & $\begin{array}{c}3.16 \\
(1.20-8.20)\end{array}$ & 0.7 & 1.9 & $\begin{array}{c}2.71 \\
(0.42-15.28)\end{array}$ & 2.3 & $\begin{array}{c}3.29 \\
(0.55-18.19)\end{array}$ \\
\hline $18-19$ & $2016 / 2017$ & 610 & 16.4 & 19.6 & $\begin{array}{c}1.20 \\
(0.79-1.81)\end{array}$ & 15.7 & $\begin{array}{c}0.96 \\
(0.61-1.49)\end{array}$ & 2.4 & 4.5 & $\begin{array}{c}1.88 \\
(0.64-5.49)\end{array}$ & 3.9 & $\begin{array}{c}1.63 \\
(0.54-4.88)\end{array}$ \\
\hline
\end{tabular}

95\%Cl: 95\% confidence interval; PR: prevalence ratio.

* Includes overweight and obesity;

** Reference category. 
In the first Pelotas cohort (1982), excess weight prevalence increased 1.62 times (95\%CI: 1.51-1.73), from 18-19 to 22 years, a result similar to the increase observed for obesity (PR = 1.76; 95\%CI: 1.53 2.02). From 22 to 30 years, the prevalence of excess weight doubled ( $\mathrm{PR}=1.99 ; 95 \% \mathrm{CI}$ : $1.91-2.08)$ and of obesity almost tripled ( $\mathrm{PR}=2.76$; $95 \% \mathrm{CI}: 2.53-3.02)$. It is noteworthy that at 30 years of age, $57.5 \%$ of the individuals presented excess weight and 22.9\% were obese (Table 1 and Figure 1b; Data from PR and 95\%CI not shown in table).

In the second cohort (1993), from 11 to 18 years, the prevalence of excess weight increased 1.13 times (95\%CI: 1.08-1.19), and of obesity, 1.53 times (95\%CI: 1.36-1.71). From 18-19 to 22 years, the prevalence of excess weight increased 1.60 times (95\%CI: 1.54-1.68), while the prevalence of obesity almost doubled ( $\mathrm{PR}=1.87$; 95\%CI: 1.71-2.04). At 22 years, $44.2 \%$ of participants had excess weight and 16.9\% were obese (Table 1 and Figure 1; data from PR and 95\%CI not shown in table).

In the third cohort (2004), at 11 years, the prevalence of excess weight was $37.2 \%$ and that of obesity was $13.2 \%$ (Table 1 and Figure 1).

Comparing the cohorts with follow-ups in similar age groups, at 11 and 18-19 years, the younger the cohort, the higher the prevalence of excess weight and obesity. In addition, the greatest increase was observed in obesity prevalence, rather than in excess weight prevalence, at all follow-up intervals (Table 2).

In the 1982 cohort, lower prevalences of excess weight were observed among women when compared to men at 22 years and 30 years. In the 1993 cohort, at 18-19 and 22 years, obesity prevalence was $23 \%$ and $39 \%$, higher in females than in males (Table 3 ).

In the 1982 and 1993 cohorts, at age 22, a lower prevalence of obesity was observed among individuals in the third tertile compared to the first income tertile. In the 1993 and 2004 cohorts, at 11 years, the higher the income tertile, the greater the excess weight prevalence. In the 1982 cohort, at 30 years, a higher excess weight prevalence was observed among individuals in the second income tertile when compared to those in the first tertile. In addition, obesity prevalence in the 1993 cohort at 11 years was $64 \%$ higher among individuals in the third tertile compared to the first income tertile (Table 4).

\section{Prevalence of excess weight and obesity - São Luís cohort}

From 7-9 to 18-19 years of age, excess weight prevalence increased 3.04 times (95\%CI: 2.19-4.24), whereas obesity prevalence almost doubled ( $\mathrm{PR}=1.87$; 95\%CI: 1.09-3.53) (Table 1 and Figure 1; PR and $95 \%$ CI not shown in table).

There were no differences in the prevalences of excess weight and obesity between sexes (Table 3). At 7-9 years of age, excess weight prevalence was 2.16 times higher among individuals in the third tertile when compared to those in the first income tertile (Table 4).

\section{Prevalence of excess weight and obesity - all cohorts}

In cohorts with a follow-up at 8 to 11 years, with the exception of the São Luís cohort, which had the lowest prevalence in this age group, the younger cohorts had higher prevalences of excess weight and obesity (Table 2).

At 18-19 years, prevalences of excess weight and obesity were also higher in the youngest cohort of Pelotas (1993) compared to the oldest (Pelotas 1982). In São Luís, however, prevalences of excess weight and obesity at 18-19 years were similar to the prevalences observed in the 1982 Pelotas cohort in the same age group, which started 15 years ago (Table 2).

At 22-25 years, except in Pelotas (1982), which had lower prevalences in that age group, there were also higher prevalences of excess weight and obesity in the youngest cohorts (Table 2).

In addition, when comparing cohorts with follow-ups at similar age groups and in close years, both at 8-11 years (in 2004/2006) and at 18-23 years (in 2015/2017), the highest prevalences of excess weight and obesity were observed in Ribeirão Preto, while São Luís had the lowest prevalences (Table 2). 
Regarding sex, there were no differences in the prevalences of excess weight and obesity up to 11 years in all cohorts. However, from the age of 22 onwards, there was a lower prevalence of excess weight among women in the oldest cohorts; and, from the age of 18 , the highest prevalence of obesity among women was observed in the younger cohorts (Table 3).

As for income, in the age range from 7-11 years in the cohorts of the three cities, the prevalence of excess weight was higher among children in the second and/or third tertile of income. On the other hand, from 22-25 years, a lower prevalence of obesity was observed among individuals in the highest income tertile (Table 4).

\section{Discussion}

The prevalences of excess weight and obesity increased in all cohorts of the evaluated cities, being higher in the younger cohorts of each city, when comparing similar age ranges. Moreover, the increase in the prevalences of obesity were greater than those of excess weight. In similar age ranges and close follow-up years, higher prevalences of excess weight and obesity were observed in Ribeirão Preto, the most developed of the three locations 7, while slightly lower prevalences were observed in Pelotas and much lower in São Luís. Men of the older cohorts presented higher prevalences of excess weight and women of the younger cohorts had higher prevalences of obesity. Adults with lower income and richer children and adolescents had the highest prevalences of excess weight and obesity.

Prevalences of excess weight and obesity increased in all cohorts of the three cities over the followups. Increases in excess weight and obesity prevalences have been consistently reported in Brazil 17 and in the world 1.

In all cohorts, increases in the prevalences of excess weight and obesity occurred with age. Similarly, data from the 2016 Risk and Protective Factors Surveillance System for Chronic Non-Comunicable Diseases Through Telephone Interview (Vigitel) survey indicate that the prevalence of obesity has increased twice from 18-24 to 25-34 years. In addition, the increase in excess weight prevalence continued until 44 years in men and up to 64 years in women 17.

São Luís presented the lowest prevalences of excess weight and obesity at all ages. This result corroborates data from Vigitel, which point São Luís as the capital with the lowest prevalences of excess weight and obesity 2,18. Despite having a very similar HDI to Pelotas 7 , this municipality has worse indicators of longevity and income (although it has better education indicators) 19,20.

Although the lowest prevalences of excess weight have been observed in São Luís, in this cohort the largest relative increases in excess weight and obesity were identified. From 7-9 to 18-19 years, excess weight prevalence increased 3.04 times. In 1994 Ribeirão Preto birth cohort, excess weight increased 1.76 times, from 9-11 to 22-23 years. In 1993 Pelotas cohort, the relative increase in excess weight prevalence was 1.13 times, from 11 to 18 years. This scenario indicates that nutritional transition in Brazil does not occur in a uniform manner, progressing at a more advanced stage in Ribeirão Preto and Pelotas and at an earlier stage in São Luís. Although these comparisons are not exactly made at the same ages and calendar years, they are still very close.

Prevalences of excess weight and obesity were higher in the younger cohorts. This result indicates that individuals in these cohorts appear to be exposed to more obesogenic environments when compared to the oldest ones. Similar results were observed by Jaacks et al. 21 in China and Robinson et al. 4 in United States. In Brazil, despite the implementation of policies and programs to encourage healthy eating and physical activity 22,23 , some factors have contributed to the changing of environments (thus making them more obesogenic), such as increase in the mean daily time spent on the internet, number of households with television sets, number of cars and per capita consumption of prepared foods and industrial mixtures 24 .

There were no differences in the prevalence of excess weight and obesity between sexes in childhood and early adolescence. However, from 18 years onwards, higher prevalences of excess weight were identified among males in the oldest cohorts and higher prevalences of obesity in female in the youngest cohorts. Those results corroborate data from national surveys 3,17 and suggest that generations of younger women had a more intense weight gain than men, leading to a shift of the BMI curve to the right. Possibly, the insertion of women in the labor market and consequently their 
greater purchasing power and decision-making at home has contributed to a lesser availability of time to care with food. This led women to choose ready-made or quick preparation of food items, with higher caloric density 25,26. Moreover, mechanization of domestic work, promoted by greater access to household appliances, has favored a reduction in energy expenditure due to carrying out household tasks, even more so by women 27.

Higher prevalences of obesity were observed among the poorest young adults (22-25 years). Although for a long time obesity has been seen as a condition associated with the socioeconomic elite, studies in the last decade have shown the shift of this morbidity to individuals belonging to the poorest economic classes, even in middle-income countries. In a study with data from national surveys from 1974 to 200928 it was observed that in men and women obesity presented greater relative annual increase in individuals of the lowest income quintile. Other study ${ }^{29}$ showed that between 1974 and 2003 the risk of obesity in Brazil was higher among poorer individuals and that, among women, the protective effect of the lower income on the development of obesity was eliminated.

Higher prevalences of excess weight and obesity in the poorest adults may be related to their food consumption. According to POF (Brazilian Household Budget Survey) of 2008-2009, consumption of fruits and salads was lower among individuals with lower income, which may contribute to the increased excess weight and obesity 30 .

Another important aspect is that health promotion actions in Brazil are still very focused on the transmission of information at the individual level, which puts groups with higher income and education in an advantageous position to avail such information 29. It is expected that groups more favored in terms of income and education may have more awareness of the risks related to excess weight and obesity to their health, as well as a greater understanding of the nutritional concepts of healthier eating 31.

From 7-11 years, the result was the opposite of that observed among adults. Children and adolescents belonging to the highest income tertiles had higher prevalence of excess weight. According to POF of 2008-2009, the group that most consumes food outside home in Brazil were richer adolescents. It is worth mentioning that food consumption outside the household is, predominantly, characterized by the intake of fast foods and sugary drinks 30 . Other studies have reported that children and adolescents with higher socioeconomic status have a worse profile of food consumption (higher intake of soft drinks, yoghurts, chocolates, breakfast cereals and modified powdered milk) ${ }^{32}$. Furthermore, lower levels of physical activity have been reported among Brazilian children and adolescents with higher socioeconomic status, contributing to the development of excess weight 33,34.

In addition, one can also consider the protective effect of school lunch offered in public schools 35. Children from lower income are more likely to study in public schools and consume school lunch. In turn, school cafeterias, more present in private schools, have been associated with increased excess weight among children, due to the predominance of ultra-processed foods or fried preparations 36 .

This study has some limitations. Follow-up losses were among such limitations; however, in order to counter this problem, inverse probability weighting has been performed. Despite high loss rates in some follow-ups, the differences between weighted and unweighted estimates were mostly small (median: 0.1\%; interquartile range: $0.3 \%$ ). Another limitation was the impossibility of separating age, period and cohort effects due to the small number of follow-ups in the same periods and ages.

Otherwise, this study has as its strong points: the data, which has been collected with high methodological rigor from the main birth cohorts in Brazil, with large sample sizes and most of them, considering a high follow-up rate. Furthermore, these cohorts are distributed in three regions with different demographic, socioeconomic and development characteristics. Cohort studies are valuable because they permit the comparison between obesity trends in the same individuals over time.

The results of the our study contribute to the analysis of the evolution of excess weight and obesity prevalence over time in three Brazilian cities, located in different regions. Differences in the prevalence of excess weight and obesity evidenced that individuals from younger cohorts are more exposed to this morbidity (especially women, in relation to obesity), as well as those who were born in the most developed city, low-income adults as well as children and adolescents belonging to families of the highest income tertile. Therefore, the results of this study indicate the need to prioritize actions aimed at younger individuals. Interventions should be conducted early in childhood and adolescence, as excess weight and obesity have advanced at faster pace in these groups. 


\section{Contributors}

C. A. Carvalho and A. K. T. C. França contributed in the conception and design of the study, analysis and interpretation of data and draft of the manuscript. E. I. S. Magalhães contributed in the analysis and interpretation of data and draft of the paper. H. Bettiol, M. A. Barbieri, V. C. Cardoso, A. Matijasevich, A. M. B. Menezes, B. L. Horta, F. C. Wehrmeister, H. Gonçalves, I. S. Santos and N. P. Lima contributed to the conception and design of the study and critical review of the intellectual content. A. A. M. Silva contributed in the conception and design of the study, analysis and interpretation of data and critical review of intellectual content.

\section{Additional informations}

ORCID: Carolina Abreu de Carvalho (00000001-7900-4642); Elma Izze da Silva Magalhães (0000-0001-9909-9861); Heloisa Bettiol (00000001-8744-4373); Marco Antonio Barbieri (00000001-8060-1428); Viviane Cunha Cardoso (00000002-2677-5600); Alicia Matijasevich (0000-00030060-1589); Ana Maria Baptista Menezes (00000002-4129-0898); Bernardo Lessa Horta (00000001-9843-412X); Fernando C. Wehrmeister (0000-0001-7137-1747); Helen Gonçalves (00000001-6470-3352); Iná S. Santos (0000-0003-12589249); Natália Peixoto Lima (0000-0002-71813717 ); Ana Karina Teixeira da Cunha França (00000002-4460-2631); Antônio Augusto Moura da Silva (0000-0003-4968-5138).

\section{Acknowledgments}

We thank the funding agencies that supported the research in the cohorts included in the study (Brazilian Graduate Studies Coordinating Board, Brazilian Public Health Association, Brazilian National Research Council, Department of Science and Technology, World Health Organization, Children's Pastoral Commision, PROEX, PRONEX, European Union, International Development Research Centre) and all participants.

\section{References}

1. NCD Risk Factor Collaboration. Trends in adult body-mass index in 200 countries from 1975 to 2014: a pooled analysis of 1698 population-based measurement studies with 19.2 million participants. Lancet 2016; 387:1377-96.

2. Departamento de Análise em Saúde e Vigilância de Doenças não Transmissíveis, Secretaria de Vigilância em Saúde, Ministério da Saúde. Vigitel Brasil 2018: Vigilância de Fatores de Risco e Proteção para Doenças Crônicas por Inquérito Telefônico. Estimativas sobre frequência e distribuição sociodemográfica de fatores de risco e proteção para doenças crônicas nas capitais dos 26 estados brasileiros e no Distrito Federal em 2018. Brasília: Ministério da Saúde; 2019.

3. Instituto Brasileiro de Geografia e Estatística. Pesquisa de Orçamentos Familiares 20082009 - antropometria e estado nutricional de crianças, adolescentes e adultos no Brasil. Rio de Janeiro: Instituto Brasileiro de Geografia e Estatística; 2010.

4. Robinson WR, Keyes KM, Utz RL, Martin CL, Yang Y. Birth cohort effects among US-born adults born in the 1980s: foreshadowing future trends in US obesity prevalence. Int J Obes 2013; 37:448-54.

5. Confortin SC, Ribeiro MRC, Barros AJD, Menezes AMB, Horta BL, Victora CG, et al. RPS Consortium of Brazilian Birth Cohorts (Ribeirão Preto, Pelotas and São Luís): history, objectives and methods. Cad Saúde Pública 2021; 37:e00093320.

6. Instituto Brasileiro de Geografia e Estatística. Estimativas da população residente no Brasil e unidades da federação com data de referência em 1o de julho de 2019. https://agenciadenoti cias.ibge.gov.br/media/com_mediaibge/arqui vos/7d410669a4ae85faf4e8c3a0a0c649c7.pdf (acessado em 28/May/2020).

7. Cardoso VC, Simoes VM, Barbieri MA, Silva AA, Bettiol H, Alves MT, et al. Profile of three Brazilian birth cohort studies in Ribeirao Preto, SP and São Luis, MA. Braz J Med Biol Res 2007; 40:1165-76.

8. Santos IS, Barros AJ, Matijasevich A, Zanini R, Cesar MAC, Camargo-Figuera FA, et al. Cohort profile update: 2004 Pelotas (Brazil) Birth Cohort Study. Body composition, mental health and genetic assessment at the 6 years follow-up. Int J Epidemiol 2014; 43:1437-37f.

9. Horta BL, Gigante DP, Gonçalves H, Motta JVS, Mola CL, Oliveira IO, et al. Cohort profile update: the 1982 Pelotas (Brazil) Birth Cohort Study. Int J Epidemiol 2015; 44:441-e.

10. Gonçalves H, Wehrmeister FC, Assunção MCF, Tovo-Rodrigues L, Oliveira IO, Murray J, et al. Cohort profile update: the 1993 Pelotas (Brazil) Birth Cohort follow-up at 22 years. Int J Epidemiol 2018; 47:1389-90e.

11. Simões VMF, Batista RFL, Alves MTSSB, Ribeiro CCC, Thomaz EBAF, Carvalho CA, et al. Saúde dos adolescentes da coorte de nascimentos de São Luís, Maranhão, Brasil, 1997/1998. Cad Saúde Pública 2020; 36:e00164519. 
12. Jelliffe DB. The assessment of the nutritional status of the community. Geneva: World Health Organization; 1966. (WHO Monograph Series, 53).

13. Ministério da Saúde. Sistema de Vigilância Alimentar e Nutricional: orientações para coleta e análise de dados antropométricos em serviços de saúde. Norma técnica do Sistema de Vigilância Alimentar e Nutricional (SISVAN). Brasília: Ministério da Saúde; 2011.

14. Cole TJ, Bellizzi MC, Flegal KM, Dietz WH. Establishing a standard definition for child overweight and obesity worldwide: international survey. BMJ 2000; 320:1240.

15. World Health Organization. Physical status: the use and interpretation of anthropometry - Report of a WHO Expert Committee. Geneva: World Health Organization; 1995. (WHO Technical Report Series, 854).

16. Departamento de Vigilância de Doenças e Agravos não Transmissíveis e Promoção da Saúde, Secretaria de Vigilância em Saúde, Ministério da Saúde. Vigitel Brasil 2016 - Vigilância de Fatores de Risco e Proteção para Doenças Crônicas por Inquérito Telefônico: Estimativas sobre frequência e distribuição sociodemográfica de fatores de risco e proteção para doenças crônicas nas capitais dos 26 estados brasileiros e no Distrito Federal em 2016. Brasília: Ministério da Saúde; 2017.

17. Secretaria de Vigilância em Saúde; Secretaria de Gestão Estratégica e Participativa; Ministério da Saúde. Vigitel Brasil 2006: vigilância de fatores de risco e proteção para doenças crônicas por inquérito telefônico. Brasília: Ministério da Saúde; 2007. (Série G. Estatística e Informação em Saúde).

18. Atlas Brasil. Altas do desenvolvimento humano no Brasil - Pelotas, RS. http://www.atlas brasil.org.br/2013/pt/perfil_m/pelotas_rs (accessed on 28/May/2020).

19. Atlas Brasil. Altas do desenvolvimento humano no Brasil - São Luís, MA. http://www. atlasbrasil.org.br/2013/pt/perfil_m/sao-luis_ ma (accessed on 28/May/2020).

20. Jaacks LM, Gordon-Larsen P, Mayer-Davis EJ, Adair LS, Popkin B. Age, period and cohort effects on adult body mass index and overweight from 1991 to 2009 in China: the China Health and Nutrition Survey. Int J Epidemiol 2013; 42:828-37.

21. Castro IRR. Desafios e perspectivas para a promoção da alimentação adequada e saudável no Brasil. Cad Saúde Pública 2015; 31:7-9.

22. Amorim T, Knuth A, Cruz D, Malta D, Reis R, Hallal P. Description of the physical activity promotion programs funded by the Brazilian Ministry of Health. Rev Bras Ativ Fís Saúde 2013;18:63-74.

23. Flores-Ortiz R, Malta DC, Velasquez-Melendez G. Adult body weight trends in 27 urban populations of Brazil from 2006 to 2016: a population-based study. PLoS One 2019; 14:e0213254.
24. Schlindwein MM, Kassouf AL. Mudanças no padrão de consumo de alimentos tempo-intensivos e de alimentos poupadores de tempo, por região no Brasil. v. 2. Brasília: Instituto de Pesquisa Econômica Aplicada; 2007.

25. Lelis CT, Teixeira KMD, Silva NMD. A inserção feminina no mercado de trabalho e suas implicações para os hábitos alimentares da mulher e de sua família. Saúde Debate 2012; 36:523-32.

26. Covolan NT, Carvalho MG. Tempo e tecnologia: o espaço doméstico sob a ótica das/os pesquisadoras/es de gênero. Revista Tecnologia e Sociedade 2015; 11:36-49.

27. Conde WL, Monteiro CA. Nutrition transition and double burden of undernutrition and excess of weight in Brazil. Am J Clin Nutr 2014; 100:1617S-22.

28. Monteiro CA, Conde WL, Popkin BM. Income-specific trends in obesity in Brazil: 1975-2003. Am J Public Health 2007; 97:180812.

29. Instituto Brasileiro de Geografia e Estatística. Pesquisa de Orçamentos Familiares 20082009 - análise do consumo alimentar pessoal no Brasil. https://biblioteca.ibge.gov.br/visua lizacao/livros/liv50063.pdf (accessed on 21/ $\mathrm{Feb} / 2020)$.

30. Caprio S, Daniels SR, Drewnowski A, Kaufman FR, Palinkas LA, Rosenbloom AL, et al. Influence of race, ethnicity, and culture on childhood obesity: implications for prevention and treatment a consensus statement of shaping America's Health and the Obesity Society. Diabetes Care 2008; 31:2211-21.

31. Levy RB, Castro IRR, Cardoso LDO, Tavares LF, Sardinha LMV, GomesFS, et al. Consumo e comportamento alimentar entre adolescentes brasileiros: Pesquisa Nacional de Saúde do Escolar (PeNSE), 2009. Ciênc Saúde Colet 2010; 15 Suppl 2:3085-97.

32. Hallal PC, Bertoldi AD, Gonçalves H, Victora CG. Prevalência de sedentarismo e fatores associados em adolescentes de 10-12 anos de idade. Cad Saúde Pública 2006; 22:1277-87.

33. Matsudo VKR, Ferrari GLDM, Araújo TL, Oliveira LC, Mire E, Barreira TV, et al. Indicadores de nível socioeconômico, atividade física e sobrepeso/obesidade em crianças brasileiras. Rev Paul Pediatr 2016; 34:162-70.

34. Freitas JFB. Alimentação na escola e excesso de peso em adolescentes do Estudo de Riscos Cardiovasculares em Adolescentes - ERICA Estudo Piloto [Masters' Thesis]. Rio de Janeiro: Universidade Federal do Rio de Janeiro; 2014.

35. Rossi CE, Costa LCF, Machado MS, Andrade DF, Vasconcelos FAG. Fatores associados ao consumo alimentar na escola e ao sobrepeso/ obesidade de escolares de 7-10 anos de Santa Catarina, Brasil. Ciênc Saúde Colet 2019; 24:443-54. 


\section{Resumo}

O estudo teve como objetivo estimar as prevalências de excesso de peso e de obesidade, de acordo com sexo e renda, no Consórcio RPS (Ribeirão Preto, Pelotas e São Luís) de coorte de nascimentos brasileiros. Os participantes nas coortes de Ribeirão Preto (1978/1979 e 1994), Pelotas (1982, 1993 e 2004) e São Luís (1997/1998) foram incluídos em diferentes seguimentos a partir dos sete anos de idade. $O$ excesso de peso (sobrepeso e obesidade) $e$ a obesidade foram avaliados pelo indice de massa corporal. As maiores prevalências foram observadas em Ribeirão Preto (excesso de peso: $27,7 \%$ aos 9-11 e 47,1\% aos 22-23 anos; obesidade: 8,6\% aos 9-11 e 19,8\% aos 22-23 anos) e as menores taxas em São Luís (excesso de peso: 5,4\% aos 7-9 e 17,2\% aos 18-19 anos; obesidade: $1,8 \%$ aos $7-9$ e 3,6\% aos 18-19 anos). Quanto mais jovem a coorte, maior a prevalência de excesso de peso e de obesidade, na comparação entre grupos etários semelhantes. $O$ aumento na prevalência de obesidade foi maior que na prevalência de excesso de peso. As mulheres tiveram menor prevalencia de excesso de peso nas coortes mais velhas e maior prevalência de obesidade nas coortes mais jovens. Maiores prevalências de excesso de peso e de obesidade foram observadas nas crianças e adolescentes com renda familiar mais alta e em adultos de renda mais baixa. As diferenças nas prevalências de excesso de peso $e$ de obesidade evidenciaram que os indivíduos das coortes mais jovens estão mais expostos a essa morbidade, assim como, aqueles nascidos na cidade mais desenvolvida, os adultos de baixa renda $e$ crianças e adolescentes pertencentes a família do tercil de maior renda. Portanto, os resultados indicam a necessidade de priorizar medidas dirigidas aos indivíduos mais jovens.

Sobrepeso; Obesidade; Transição Nutricional; Prevalência

\section{Resumen}

El objetivo de este estudio fue estimar la prevalencia de sobrepeso y obesidad, según sexo e ingresos en el Consorcio RPS (Ribeirão Preto, Pelotas and São Luís) cohortes brasileñas de nascimientos. Los participantes en las cohortes de nacimiento de $\mathrm{Ri}$ beirão Preto (1978/1979 y 1994), Pelotas (1982, 1993 y 2004) y São Luís (1997/1998) fueron incluidos en diferentes seguimientos desde los 7 años de edad. El exceso de peso (sobrepeso y obesidad) fueron evaluados por el indice de masa corporal. Las prevalencias más altas se observaron en $\mathrm{Ri}$ beirão Preto (exceso de peso: 27,7\% a los 9-11 y 47,1\% a los 22-23 años; obesidad: 8,6\% a los 9-11 y 19,8\% a los 22-23 años) y los más pequeños en São Luís (exceso de peso: 5, 4 a los 7-9y 17,2\% a los 18-19 años; obesidad: 1,8\% a los 7-9y 3,6\% a los 18-19 años). Cuanto más joven fuera la cohorte, mayor era la prevalencia de exceso de peso y obesidad, cuando se comparan con grupos de edad similares. El incremento en la prevalencia de obesidad fue mayor que en la prevalencia de exceso de peso. Las mujeres tenían una prevalencia de exceso de peso más baja en las cohortes más viejas y una mayor prevalencia de obesidad en las cohortes más jóvenes. Se observaron prevalencias más altas de exceso de peso y obesidad en los niños y adolescentes con mayores ingresos, y en los adultos más pobres. Las diferencias en las prevalencias de exceso de peso y obesidad evidenciaron que las personas de las cohortes más jóvenes estaban más expuestas a esta morbilidad, al igual que aquellas que habian nacido en las ciudades más desarrolladas, adultos con bajos ingresos, además de niños y adolescentes pertenecientes a las familias del tercil de ingresos más altos. Por eso, los resultados de este estudio indican la necesidad de priorizar acciones dirigidas a las personas más jóvenes.

Sobrepeso; Obesidad; Transición Nutricional; Prevalencia
Submitted on 10/Aug/2020

Final version resubmitted on 18/Dez/2020

Approved on 04/Jan/2021 